\section{Primary lysis/necrosis of eosinophils and clinical control of asthma}

I read with interest the demonstration by Volbeda et $a l^{1}$ that clinical asthma control associates significantly with 'lower activated eosinophil numbers in the bronchial wall, yet only weakly with sputum eosinophils'. I was particularly intrigued by the online supplemental information ${ }^{1}$ : 'EPX staining showed widely spread distribution of eosinophilic granules, not necessarily in close proximity to EPX+ cells. Therefore, degranulation of eosinophils was determined by quantification of the EPX immunopositive area by computer-assisted image analysis'. This statement suggests to me that the wall eosinophils had largely been activated by lysis of eosinophils that deposits protein-laden eosinophil granules extracellularly in the diseased tissue. ${ }^{2}$

For a centennium, the occurrence of free eosinophil granules in airway tissues and sputum samples were either ignored or considered an artefact caused by sample handling. ${ }^{3}$ However, wholemount tissue preparation combined with deep tissue staining of eosinophil proteins clearly demonstrated that free eosinophil granules were a real in vivo phenomenon. ${ }^{2}$ Transmission electron microscopy further demonstrated that the granules emanated from eosinophils exhibiting evident lysis/ necrosis (chromatolysis, cell membrane rupture, etc). Importantly, the lysis of eosinophils was not preceded by apoptosis, and it happened to eosinophils with little prior piecemeal degranulation. ${ }^{2}$ Intense staining in human diseased airways tissue indicated significant release of toxic eosinophil proteins from the free granules, ${ }^{2}{ }^{3}$ a process now being explored in vitro. ${ }^{4}$ Numbers of free eosinophil granules in the airway wall have also correlated positively with disease activity in asthma and rhinitis. ${ }^{3}$ These data, together with the current report, ${ }^{1}$ support the notion that eosinophil lysis releasing granules (plus many biologically active nuclear/ cytosolic molecules!), may have a causative role in eosinophilic airways diseases.
Transepithelial migration of eosinophils (rather than apoptosis/phagocytosis mechanisms) will eliminate these cells from the diseased bronchial wall. Hence, cell numbers in the lumen may not mirror cell numbers in the wall during important phases of bronchial mucosal inflammation. ${ }^{5}$ In agreement, Volbeda et al ${ }^{1}$ found that activated eosinophils in bronchial biopsies better than eosinophil numbers in sputum reflect the level of disease control. It would be of interest to learn about location of the free granules; for instance, any association with epithelial fragility in uncontrolled asthma ${ }^{1}$ ? The possibility that ongoing lysis of eosinophils is important for lack of asthma control $^{1}$ underscores the need to unravel mechanisms involved in lysis of eosinophils and discover their pharmacological regulation.

\section{Carl Persson}

Department of Clinical Pharmacology, Laboratory Medicine, University Hospital Lund, Lund, Sweden

Correspondence to Dr Carl Persson, Department of Clinical Pharmacology, Laboratory Medicine, University Hospital Lund, Lund S22185, Sweden;

carl.persson@med.lu.se

Funding None.

Competing interests None.

Patient consent Obtained.

Provenance and peer review Not commissioned; internally peer reviewed.

To cite Persson C. Thorax 2013;68:295.

Received 1 November 2012

Accepted 22 November 2012

Published Online First 15 December 2012

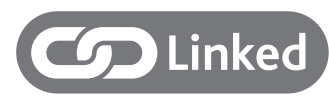

- http://dx.doi.org/10.1136/thoraxjnl-2012-203023

Thorax 2013;68:295.

doi:10.1136/thoraxjnl-2012-202955

\section{REFERENCES}

1 Volbeda F, Broekma M, Lodewijk ME, et al. Clinical control of asthma associates with measures of airway inflammation. Thorax 2012;68:19-24.

2 Persson CGA, Erjefält JS. Eosinophil lysis and free granules: an in vivo paradigm for cell activation and drug development. Trends Pharmacol Sci 1997;18:117-23.

3 Persson CGA. Centennial notions of asthma as an eosinophilic, desquamative, exudative, and steroid-sensitive disease. Lancet 1997;350:1021-4.

4 Neves JS, Weller PF. Functional extracellular eosinophil granules: novel implications in eosinophil immunobiology. Curr Opin Immunol 2009;21:694-9.

5 Persson CG, Uller L. Transepithelial migration of leucocytes: inflicting, reflecting or resolving airway inflammation? Thorax 2010;65:111-5. 